\title{
Aciertos y fallas en la implementación de la Política de Mujeres y Equidad de Género de Bogotá*
}

\author{
Successes and failures in the implementation \\ of the Policy for Women and Gender Equality \\ in Bogota
}

Génesis Fernanda Barón Mesa
Janeth Patricia Muñoz Eraso

Recibido: 15 de julio de 2015

Aprobado: 8 de febrero de 2016

Disponible en línea: 30 de junio de 2016

\section{Resumen}

Para explicar los aciertos y las fallas en la implementación de las políticas públicas, se analiza el caso de la Política Pública de Mujeres y Equidad de Género de Bogotá en el periodo 2004-2013. Tomando como marco de análisis la fase de implementación de las políticas públicas, se demuestra que esta Política ha logrado avances importantes en el reconocimiento, la garantía y la restitución de los derechos de las mujeres y crea espacios relevantes para el fortalecimiento de los procesos de participación y organización de la población femenina, pero también indica

\section{Abstract}

To explain the successes and failures in the implementation of public policy, we analized the case of the Policy for Women and Gender Equality in Bogota in the period between 2004 and 2013. Taking as the framework for analysis the implementation phase of public policy, we show that this policy has made significant progress in recognizing, guaranteeing and restituting the rights of women and creates relevant spaces for strengthening participatory processes and organization of the female population, but also indicates failures related to the difficulty of

doi:10.11144/Javeriana.papo21-1.afip

* Este artículo es un producto parcial de la tesis "La implementación de la Política Pública de Mujeres y Equidad de Género en el Distrito Capital 2004-2013: ¿formalismos o realidad?, desarrollada por Génesis Fernanda Barón para optar al título de Politóloga con Énfasis en Gestión Pública de la Pontificia Universidad Javeriana de Bogotá y dirigida por la profesora Janeth Patricia Muñoz Eraso.

** Politóloga con Énfasis en Gestión Pública. Correo electrónico: ferbame18@yahoo.es

*** Magíster en Desarrollo Rural por la Pontificia Universidad Javeriana de Bogotá y Doctora en Ciencias Sociales con Especialización en Estudios Políticos por Facultad Latinoamericana de Ciencias Sociales, Ecuador. Consultora y profesora de la Carrera de Ciencia Política de Pontificia Universidad Javeriana de Bogotá. Correo electrónico: janeth.munoz@javeriana.edu.co 
fallas relacionadas con la dificultad de introducir el enfoque de género y derechos en los procesos de planeación e institucionalización de algunos sectores de la Administración distrital, así como dificultades en la asignación de presupuestos específicos para su puesta en marcha.

\section{Palabras clave}

política pública; implementación; instrumentos; género; equidad; mujer; Bogotá

\section{Cómo citar este artículo:}

Barón Mesa, G. F. y Muñoz Eraso, J. P. (2016). Aciertos y fallas en la implementación de la Política de Mujeres y Equidad de Género de Bogotá. Papel Político, 21(1), 101-120. http:// dx.doi.org/10.11144/Javeriana.papo21-1.afip introducing the gender and rights approach in planning processes and institutionalization of some sectors of the district administration, as well as difficulties in allocating specific budgets for its implementation.

\section{Keywords}

public policy implementation; instruments; gender equality; woman; Bogotá 


\section{Introducción}

La población femenina cumple un papel fundamental en las esferas públicas y privadas de Colombia. Pero así mismo, es innegable la existencia de desigualdades entre hombres y mujeres en el acceso a estos espacios. Los avances logrados en cuanto a las reivindicaciones de las mujeres respecto de su rol en la sociedad no han sido suficientes, porque persiste la desigualdad entre los géneros, reflejada, entre otras, en la falta de oportunidades reales para que las mujeres accedan en igualdad de condiciones y oportunidades a las diferentes esferas políticas, económicas y sociales. Según los datos de la participación política de las mujeres en el Congreso de la República, durante los últimos trece años la desigualdad se mantiene, porque no ha superado $12 \%$ de escaños ocupados (ONU, 2012). Tal situación, según el Informe del Observatorio de Igualdad de Género de América Latina y el Caribe (2012) de la ONU, ubica al país en los últimos lugares de Latinoamérica en cuanto a la representación femenina en los Congresos nacionales.

Del mismo modo, las mujeres han sido blanco de vulneración y maltrato físico, verbal y psicológico, principalmente por parte de los hombres, tal como lo señalan las cifras relacionadas con la violencia intrafamiliar y de feminicidio reportadas en las comisarías de familia de Bogotá, que evidencian 37040 casos de violencia intrafamiliar, de los cuales 76 \% fueron mujeres y $24 \%$ hombres (Pineda, 2007). O los actos de feminicidio en Bogotá, en los que una mujer es asesinada cada tres días. ${ }^{1}$ Esta situación refleja la fuerte problemática en la que han estado inmersas las mujeres colombianas, y se ha convertido en referente importante para que los diferentes Gobiernos formulen y pongan en marcha un corpus normativo para tratar de solucionar esos inconvenientes. Así lo muestran, por ejemplo, los lineamientos normativos y jurídicos en los ámbitos nacional y distrital plasmados en el Decreto 519 de 2003, la Ley 051 de 1981, la Ley 248 de 1995, el Decreto 1182 de 1999 y el Decreto 166 de 2010.

No obstante, en la práctica, las intenciones gubernamentales se han quedado en formalismos, es decir, en la consagración legal de los derechos de las mujeres o en la mera enunciación de los lineamientos de las políticas públicas, sin lograr contribuir en efecto a dar soluciones contundentes para el desarrollo integral y el empoderamiento de la población femenina. Así lo demostró Muñoz (2003, p. 98) al analizar la política para el desarrollo de la mujer rural de 1994 en Colombia y encontrar que esta se dio de manera parcial y poco efectiva, en la medida en que su aplicación no respondió a un enfoque de equidad de género, previsto en la política, sino al discurso "mujer en el desarrollo", en el que predominó una concepción asistencialista e instrumental del

${ }^{1}$ Así lo concluye una investigación de la Secretaría Distrital de la Mujer que registra 1246 mujeres asesinadas entre 2004 y 2012 (Secretaría Distrital de la Mujer, 2013d). 
rol de las mujeres en la producción de alimentos, en la generación de ingresos y en la distribución intrafamiliar de los beneficios de los programas de desarrollo.

En este contexto, este artículo analiza la Política Pública de Mujeres y Equidad de Género de Bogotá en el periodo 2004-2013 e identifica si su proceso de implementación ha sido coherente con la directriz trazada en la política o si se ha quedado solo en formalismos. En la primera parte, se describe el marco analítico y el método de la investigación. En la siguiente sección, se muestran y discuten los resultados, al resaltar las realizaciones, las fallas y el modelo de implementación identificado. Finalmente, se presentan las conclusiones de este estudio de caso.

\section{Marco de análisis}

La lucha contra la desigualdad de género con la Política Pública de Mujeres y Equidad de Género de Bogotá pretende un igual acceso y control por parte de mujeres y hombres sobre los bienes, servicios y recursos de Bogotá, teniendo en cuenta las diferencias de género en cuanto a intereses, necesidades y demandas (Decreto 166 de 2010). Esta política es un caso ejemplar de una política pública, cuya implementación produce fallas, debido a la falta de coherencia entre lo estipulado en el documento formal y lo puesto en práctica. Para explicar este proceso, se procedió a un análisis de la implementación, porque es una fase de una política pública "durante la cual se generan actos y efectos a partir de un marco normativo de intenciones, de textos o de discursos” (Meny y Thoenig, 1992, p. 159).

Los diversos modelos de análisis de la implementación se constituyen, como lo diría Hargrove (1975), citado por Roth (2009, p. 109), en el "lazo entre el proceso de decisión de una política y su proceso de ejecución”. Entre ellos, los enfoques top-down y bottom-up son los más tradicionales, porque van en función de la concepción utilizada para su realización práctica. Para Meny y Thoenig (1992), citados por Roth (2009), el primero corresponde a la concepción tradicional del trabajo administrativo, que se desarrolla de arriba (top) hacia abajo (down) o del centro a la periferia, enfoque que presupone una clara separación entre la formulación y la decisión de la política, por un lado, y por otro, la implementación de las decisiones. El enfoque bottom-up toma la dirección opuesta, de abajo hacia arriba, se trata de partir de los comportamientos concretos en el nivel donde existe el problema para construir la política pública poco a poco, con reglas, procedimientos y estructuras organizativas por medio de un proceso ascendente o por retroceso en vez de descendente.

En este marco, el análisis de un gran número de procesos de implementación ha llevado a debatir acerca de las condiciones necesarias para que se dé una implementación eficaz y eficiente de las políticas. Al respecto, Sabatier y Mazmanian (1979), citados por Roth (2009), proponen el enfoque de gestión pública como guía para aumentar las posibilidades 
de una implementación más exitosa de las políticas públicas. Así, identifican las siguientes cinco condiciones que los legisladores y los diseñadores de políticas públicas tendrían que tomar en cuenta si buscan cumplir con los objetivos de una política:

- El programa debe estar fundamentado en una teoría sólida relacionada con el cambio de comportamiento del público necesario para la realización de los objetivos.

- La ley o la decisión política debe contener directrices de actuación no ambiguas y debe estructurar el proceso de implementación de manera que maximice la probabilidad de que los destinatarios se comporten según lo previsto.

- Los dirigentes o responsables de los entes encargados de la implementación deben disponer de capacidades políticas y de gestión importantes y sentirse comprometidos con los objetivos de la ley.

- El programa debe disponer de apoyos activos durante todo el proceso de implementación por parte de grupos organizados de electores y por algunos legisladores clave. La actitud debe ser neutral o favorable.

- La prioridad relativa de los objetivos legales no se encuentra socavada significativamente con el paso del tiempo por la aparición de políticas públicas conflictivas o por cambios en las condiciones socioeconómicas que debilitan los fundamentos técnicos de la teoría o el apoyo político que la respalda.

\section{Método}

El caso de la Política Pública de Mujeres y Equidad de Género en el periodo 2004-2013 se escogió porque a partir de 2004 se da la formulación y puesta en marcha del Plan de Igualdad de Oportunidades para la Equidad de Género 2004-2016 en Bogotá, que posteriormente marca la pauta para la formulación e implementación de esta política. Va hasta 2013, porque en este año se dan los primeros informes de resultados por parte de la Secretaría Distrital de la Mujer, organismo creado en 2012 para implementar la Política.

La recolección de información se realizó mediante fuentes secundarias y primarias. En las fuentes secundarias, se examinaron los documentos legales y los informes técnicos emitidos por la Secretaría Distrital de la Mujer. Las fuentes primarias consisten en entrevistas semiestructuradas, dirigidas a funcionarias clave en el proceso de implementación de la Política, ${ }^{2}$ responsables de las Casas de Igualdad de Oportunidades (CIO) ${ }^{3}$

\footnotetext{
${ }^{2}$ Los criterios de selección para los funcionarios clave en el proceso de implementación de la Política Pública de Mujeres y Equidad de Género fueron: funcionarios involucrados directamente en su proceso de implementación, funcionarios con un trabajo continuo (mayor número de años) frente a su proceso de implementación, funcionarios de la Secretaría Distrital de la Mujer, actual organismo encargado de dirigir, coordinar, articular y ejecutar las etapas de su implementación, seguimiento y evaluación.

${ }^{3}$ Son su instrumento primordial de territorialización.
} 
de las localidades de Teusaquillo y Usaquén ${ }^{4}$ y a funcionarias de la Secretaría Distrital de la Mujer. Asimismo, se entrevistaron a mujeres destinatarias de la Política, ${ }^{5}$ que se beneficiaron de las $\mathrm{CIO}$ de dichas localidades. La información recolectada se sistematizó y examinó teniendo en cuenta cinco tópicos: los antecedentes, la implementación, los logros, las fallas y el modelo de implementación de la Política.

\section{Análisis de resultados}

La Política Pública de Mujeres y Equidad de Género de Bogotá incorpora un amplio repertorio de estrategias e instrumentos, encaminados a la atención de la población femenina y al fomento de la equidad de género. La implementación de esta Política tiene como antecedentes la directriz internacional que marca las pautas para la puesta en marcha de acciones afirmativas en pro de las mujeres y la igualdad de géneros en el país.

\section{Antecedentes: acciones y normativa}

La Política Pública de Mujeres y Equidad de Género surge como producto de diversos eventos (cuadro 1), que desde el escenario internacional se han promovido para el reconocimiento de las mujeres como sujetos de derechos, entre ellos, la Convención de las Naciones Unidas para la Eliminación de todas las formas de Discriminación contra la Mujer, celebrada en 1979, que produjo acciones concretas para mejorar las condiciones de vida de las mujeres. En la década de 1990, tres acontecimientos importantes marcaron la directriz en el ámbito internacional de las políticas en pro de la igualdad de género y autonomía de la mujer: la Conferencia Mundial sobre Población y Desarrollo (1994), la Convención Interamericana para Prevenir, Sancionar y Erradicar la Violencia contra la Mujer "Convención de Belem do Para” (1994) y la Declaración y la Plataforma de Acción de Beijing (1995). A esto se une la contribución de los movimientos feministas a la reivindicación de los derechos de las mujeres y al fortalecimiento del marco normativo e institucional en pro de ellas.

Estos acontecimientos han producido un corpus normativo que reafirma y reconoce la importancia de los derechos de las mujeres en diferentes ámbitos de la esfera pública y privada, los cuales se han incorporado en las agendas gubernamentales de varios países.

\footnotetext{
${ }^{4}$ La selección de las localidades de Teusaquillo y de Usaquén se hizo por los costos en la investigación, por la facilidad de acceso a información, por la seguridad del investigador y porque las dos CIO en dichas localidades fueron creadas hace más de cuatro años, lo cual facilitó el análisis de su implementación.

${ }^{5}$ Estas mujeres se seleccionaron teniendo en cuenta que estén actualmente siendo beneficiarias de las CIO, que lleven más de tres años haciendo uso de las CIO y que sean beneficiarias de las CIO de las localidades de Teusaquillo y Usaquén.
} 


\section{Cuadro 1. Acciones internacionales en pro de los derechos de las mujeres}

\begin{tabular}{|c|c|}
\hline Iniciativa & Tema o contenido \\
\hline $\begin{array}{l}\text { Convención de las Naciones Unidas } \\
\text { para la Eliminación de Todas las } \\
\text { Formas de Discriminación contra la } \\
\text { Mujer (1979) }\end{array}$ & $\begin{array}{l}\text { Garantizar a la mujer el ejercicio y el goce de los } \\
\text { derechos humanos y las libertades fundamentales en } \\
\text { igualdad de condiciones con el hombre. }\end{array}$ \\
\hline $\begin{array}{l}\text { Declaración de la Asamblea } \\
\text { General de las Naciones Unidas } \\
\text { y el Programa de Acción de } \\
\text { la Conferencia Mundial sobre } \\
\text { Derechos Humanos (1993) }\end{array}$ & $\begin{array}{l}\text { Se da relevancia a los derechos a la educación, la } \\
\text { capacitación y la información pública como esenciales } \\
\text { para promover y lograr relaciones estables y armoniosas } \\
\text { entre comunidades. }\end{array}$ \\
\hline $\begin{array}{l}\text { Conferencia Mundial sobre } \\
\text { Población y Desarrollo (1994) }\end{array}$ & $\begin{array}{l}\text { Muestra que es preciso que mujeres y hombres } \\
\text { participen e intervengan por igual en la vida productiva } \\
\text { y reproductiva, incluida la división de responsabilidades } \\
\text { en cuanto a la crianza de los hijos y al mantenimiento } \\
\text { del hogar, así como el mejoramiento de la condición y } \\
\text { autonomía de la mujer. }\end{array}$ \\
\hline $\begin{array}{l}\text { Convención Interamericana para } \\
\text { Prevenir, Sancionar y Erradicar } \\
\text { la Violencia contra la Mujer } \\
\text { "Convención de Belem do Para" } \\
\text { (1994) }\end{array}$ & $\begin{array}{l}\text { Proteger los derechos de la mujer y eliminar las } \\
\text { situaciones de violencia que las afecte. Establece que } \\
\text { toda mujer tiene derecho a una vida libre de violencia } \\
\text { tanto en el ámbito público como en el privado, y toda } \\
\text { mujer podrá ejercer libre y plenamente sus derechos } \\
\text { civiles, políticos, económicos, sociales y culturales. }\end{array}$ \\
\hline $\begin{array}{l}\text { La Declaración y la Plataforma de } \\
\text { Acción de Beijing (1995) }\end{array}$ & $\begin{array}{l}\text { Eliminar todos los obstáculos que dificultan la } \\
\text { participación activa de las mujeres y en pie de igualdad } \\
\text { en el proceso de adopción de decisiones en las esferas } \\
\text { económica, social, cultural y política. }\end{array}$ \\
\hline $\begin{array}{l}\text { Cumbre del Milenio de las Naciones } \\
\text { Unidas (2000) }\end{array}$ & $\begin{array}{l}\text { Establecer los Objetivos de Desarrollo del Milenio, entre } \\
\text { ellos, el objetivo } 3 \text { promueve la igualdad de género y la } \\
\text { autonomía de la mujer. }\end{array}$ \\
\hline $\begin{array}{l}\text { Consejo de Seguridad de las } \\
\text { Naciones Unidas }\end{array}$ & $\begin{array}{l}\text { Mediante la Resolución } 1325 \text { de } 2000 \text {, se aplican las } \\
\text { disposiciones del derecho internacional humanitario } \\
\text { que protejan los derechos de las mujeres y las niñas } \\
\text { durante y después de los conflictos, así como incorporar } \\
\text { una perspectiva de género en las operaciones de } \\
\text { mantenimiento de la paz. }\end{array}$ \\
\hline
\end{tabular}

Fuente: Elaboración propia.

En Colombia, la directriz internacional se convierte en el marco de referencia para el diseño de políticas sociales que apuntan a mejorar la situación de las mujeres en los ámbitos nacional, departamental y distrital, como en el caso de Bogotá. ${ }^{6}$

\footnotetext{
${ }^{6}$ Bogotá cuenta con una población aproximada de 6778691 personas, de las cuales 353822 son mujeres, que representan $52.2 \%$ de la población, frente a 3240469 hombres, que representan $47.8 \%$ (DANE, 2005), lo cual
} 
En el ámbito nacional, el ejercicio de la ciudadanía de la mujer inicia con el Acto Legislativo 03 de 1954, por el cual se otorga a la mujer el derecho activo y pasivo del sufragio, lo cual representa un avance fundamental en la legitimidad de la democracia colombiana, al reconocer a la mujer como ciudadana de derechos con capacidad de elegir y ser elegida. A partir de ese momento, se fortalece el marco normativo encaminado a resaltar el papel que cumple la mujer en los diferentes escenarios de la vida social, económica, cultural y política del país (cuadro 2).

\section{Cuadro 2. Normativa nacional en pro de los derechos de las mujeres}

\begin{tabular}{|l|l|}
\hline \multicolumn{1}{|c|}{ Iniciativa } & \multicolumn{1}{c|}{ Tema o contenido } \\
\hline $\begin{array}{l}\text { Decreto } 2820 \text { de } \\
\text { Ley } 051 \text { de } 1981\end{array}$ & $\begin{array}{l}\text { Se otorgan iguales derechos y obligaciones a las mujeres y a los varones } \\
\text { en relación con su maternidad y paternidad. }\end{array}$ \\
\hline $\begin{array}{l}\text { El Estado colombiano asume como injusticia la desigualdad de género, } \\
\text { se compromete a otorgar un trato igualitario a hombres y mujeres y a } \\
\text { sancionar cualquier tipo de práctica que perpetúe esta desigualdad. }\end{array}$ \\
\hline $\begin{array}{l}\text { Ley } 248 \text { de } 1995 \\
1999\end{array}$ & $\begin{array}{l}\text { Adoptar políticas orientadas a prevenir, sancionar y erradicar la violencia } \\
\text { contra la mujer. }\end{array}$ \\
\hline Ley 581 de 2000 & $\begin{array}{l}\text { Transforma la Dirección Nacional para la Equidad de la Mujer, en la } \\
\text { Consejería Presidencial para la Equidad de la Mujer, con el objeto de } \\
\text { garantizar los derechos humanos integrales e interdependientes de las } \\
\text { mujeres y la igualdad de género. }\end{array}$ \\
\hline Ley 823 de 2003 & $\begin{array}{l}\text { Promover la participación de la mujer en todos los niveles de las ramas y } \\
\text { demás órganos del poder público. }\end{array}$ \\
\hline $\begin{array}{l}\text { Garantizar la equidad y la igualdad de oportunidades para las mujeres en } \\
\text { los ámbitos público y privado. Dentro de esta ley se promueve el diseño y } \\
\text { la puesta en marcha de la política de mujer y géneros de Bogotá. }\end{array}$ \\
\hline Ley 1257 de 2008 & $\begin{array}{l}\text { Adopta normas que permitan garantizar para todas las mujeres una vida } \\
\text { libre de violencia. }\end{array}$ \\
\hline Decreto 1930 de & $\begin{array}{l}\text { Adopta la Política Pública Nacional de Equidad de Género para las } \\
\text { Mujeres y se crea una Comisión Intersectorial para su implementación } \\
\text { que garantice derechos de las mujeres desplazadas y derecho a una vida } \\
\text { libre de violencias. }\end{array}$ \\
\hline
\end{tabular}

Fuente: Elaboración propia.

En el ámbito distrital, a partir de la década de 1990 en Bogotá, se incorporaron estrategias e instrumentos más contundentes en favor de las mujeres. Así, por ejemplo, en los planes de desarrollo de los Gobiernos de Jaime Castro, Antanas Mockus y Enrique Peñalosa, aparecen acciones puntuales en temas relacionados con educación sexual, violencia de género, inclusión laboral, entre otros (cuadro 3).

muestra que las mujeres son más de la mitad de la población. Bogotá está conformada por 20 localidades y se caracteriza por ser una urbe donde confluyen diferentes dinámicas políticas, sociales, culturales y económicas. 


\section{Cuadro 3. Planes de desarrollo de Bogotá y su incorporación del tema de género 1992-2016}

\begin{tabular}{|c|c|c|c|c|}
\hline Administración & Periodo & \begin{tabular}{|c|} 
Plan de \\
Desarrollo
\end{tabular} & Estrategias & Instrumento \\
\hline Jaime Castro & 1992-1994 & $\begin{array}{l}\text { Prioridad } \\
\text { Social }\end{array}$ & $\begin{array}{l}\text { - Prevención del cáncer } \\
\text { cérvico-uterino } \\
\text { - Atención materno-infantil } \\
\text { - Protección a las mujeres } \\
\text { gestantes y lactantes y a } \\
\text { sus hijos }\end{array}$ & $\begin{array}{l}\text { Programas de educación } \\
\text { sexual para prevenir el } \\
\text { sida y las enfermedades de } \\
\text { transmisión sexual }\end{array}$ \\
\hline Antanas Mockus & 1995-1997 & $\begin{array}{l}\text { Formar } \\
\text { Ciudad }\end{array}$ & $\begin{array}{l}\text { - Atención de la violencia } \\
\text { intrafamiliar }\end{array}$ & Comisarías de Familia \\
\hline Enrique Peñalosa & $1998-2000$ & $\begin{array}{l}\text { Por la } \\
\text { Bogotá que } \\
\text { Queremos }\end{array}$ & $\begin{array}{l}\text { - Atención a discriminación } \\
\text { laboral femenina } \\
\text { - Denuncia a delitos por } \\
\text { violencia hacia las mujeres }\end{array}$ & $\begin{array}{l}\text { Acuerdos } 11 \text { y } 17 / 1998 \\
\text { dirigidos a brindar atención } \\
\text { integral a las mujeres jefas } \\
\text { de hogar }\end{array}$ \\
\hline Antanas Mockus & $2001-2003$ & $\begin{array}{l}\text { Bogotá para } \\
\text { Vivir Todos } \\
\text { del Mismo } \\
\text { Lado }\end{array}$ & $\begin{array}{l}\text { - Incluir perspectiva de } \\
\text { género en los programas } \\
\text { del distrito que incluirán a } \\
\text { la mujer como gestora }\end{array}$ & $\begin{array}{l}\text { - Programa Cartas de } \\
\text { Navegación para la política } \\
\text { social *Lineamientos de } \\
\text { política social para las } \\
\text { mujeres } \\
\end{array}$ \\
\hline $\begin{array}{l}\text { Luis Eduardo } \\
\text { Garzón }\end{array}$ & $2004-2008$ & $\begin{array}{l}\text { Bogotá sin } \\
\text { Indiferencia }\end{array}$ & $\begin{array}{l}\text { - Avanzar en el } \\
\text { reconocimiento, garantía y } \\
\text { restitución de los derechos } \\
\text { humanos de las mujeres } \\
\text { - Fomentar el aprendizaje } \\
\text { de la igualdad y la equidad }\end{array}$ & $\begin{array}{l}\text { Plan de Igualdad de } \\
\text { Oportunidades para la } \\
\text { Equidad de Género } \\
\text { - Programa Bogotá } \\
\text { con Igualdad de } \\
\text { Oportunidades } \\
\text { - Casas de Igualdad de } \\
\text { Oportunidades } \\
\text { - Política Pública de Mujer y } \\
\text { Géneros } \\
\end{array}$ \\
\hline Samuel Moreno & $2008-2012$ & $\begin{array}{l}\text { Bogotá } \\
\text { Positiva } \\
\text { para Vivir } \\
\text { Mejor }\end{array}$ & $\begin{array}{l}\text { - Acciones contundentes } \\
\text { para reivindicar los } \\
\text { derechos sociales, } \\
\text { políticos, económicos, } \\
\text { culturales de las mujeres }\end{array}$ & $\begin{array}{l}\text { - Decreto } 166 \text { de } 2010 \\
\text { Programa Bogotá Positiva } \\
\text { con las mujeres y la } \\
\text { equidad de género }\end{array}$ \\
\hline Gustavo Petro & $2012-2016$ & $\begin{array}{l}\text { Bogotá } \\
\text { Humana }\end{array}$ & $\begin{array}{l}\text { - Mecanismos de apoyo } \\
\text { a las mujeres y su } \\
\text { autonomía }\end{array}$ & $\begin{array}{l}\text { - Secretaría Distrital de la } \\
\text { Mujer } \\
\text { - Programa Bogotá } \\
\text { Humana con igualdad de } \\
\text { oportunidades y equidad } \\
\text { de género }\end{array}$ \\
\hline
\end{tabular}

Fuente: Elaboración propia.

El enfoque de género y la equidad de género emergieron por primera vez como política general con el Plan de Desarrollo Bogotá para Vivir Todos del Mismo Lado (2001-2003), en el segundo Gobierno del alcalde Antanas Mockus, tal como lo indica Fuentes (2007) al señalar que en esta Administración "los programas del Distrito tendrán perspectiva de género e incluirán a la mujer como gestora”. Estos avances se reafirmaron con el Plan de Desarrollo Distrital Bogotá sin Indiferencia de Luis Eduardo Garzón (2004-2008), que puso en marcha cuatro importantes instrumentos de política, que dan el sustento 
normativo y permiten avanzar en el enfoque de derechos específicos para las mujeres en el distrito capital, a saber: 1) el Plan de Igualdad de Oportunidades para la Equidad de Género 2004-2016, 2) el Programa Bogotá con Igualdad de Oportunidades, 3) las CIO y 4) la Política Pública de Mujer y Géneros. ${ }^{7}$

El Plan de Igualdad de Oportunidades para la Equidad de Género ${ }^{8}$ se creó mediante el Acuerdo 091 de 2003 para "reconocer, garantizar y restablecer los derechos de las mujeres, transformando las situaciones injustas y evitables de discriminación, de tal forma que la ciudad avance hacia la igualdad de oportunidades y la equidad de género”. Este Plan se planificó para un horizonte de doce años (2004-2016) y se estructura alrededor de seis derechos. ${ }^{9}$ Es la base para la formulación y puesta en marcha en 2004 de la Política Pública de Mujer y Géneros en el distrito, la cual posteriormente toma el nombre de Política Pública de Mujeres y Equidad de Género, institucionalizada formalmente en 2010.

Adicional al Plan de Igualdad de Oportunidades para la Equidad de Género, uno de los logros más importantes del Gobierno de Garzón fue la creación de las primeras $\mathrm{CIO},{ }^{10}$ que surgieron como producto del proceso de construcción colectiva de la política. En este marco, se fortaleció el movimiento social de mujeres para apoyar la campaña política de Garzón y hacer incidencia en la agenda pública. Así lo señala una de las funcionarias de la actual administración:

Uno de los elementos más importantes de esta política pública es que no ha sido un ejercicio de formulación en escritorio y una puesta en marcha meramente instrumental e institucional, sino que hay un sustrato político y de dinámicas sociales detrás de la misma política (entrevista con Carlota Alméciga, 8 de octubre de 2013).

Las CIO propician espacios para el fortalecimiento de los procesos de participación y organización de las mujeres, permiten la difusión de sus derechos y la promoción de acciones relacionadas con cada uno de los derechos priorizados en el Plan de Igualdad

\footnotetext{
${ }^{7}$ Desde enero de 2004 hasta mayo de 2010, la Política fue conocida como "Mujer y Géneros". A partir del 4 de mayo de 2010, con la expedición del Decreto 166 de 2010, se denomina Mujeres y Equidad de Género.

${ }^{8}$ Instrumento orientado a disminuir progresivamente la discriminación, exclusión y subordinación que enfrentan las mujeres de todas las edades, culturas, orientaciones sexuales, condiciones étnico-raciales, situaciones socioeconómicas vulnerables o de desplazamiento y capacidades motoras, visuales, auditivas, psicológicas o cognitivas diferentes, que habitan en Bogotá (Alcaldía Mayor de Bogotá, 2005).

${ }^{9}$ 1) Derecho a una vida libre de violencias, 2) derecho a la participación y la representación de las mujeres, 3) derecho al trabajo en condiciones de igualdad y dignidad, 4) derecho a la salud plena, 5) derecho a la educación con equidad y 6) derecho a una cultura libre de sexismo.

${ }^{10}$ Las COI son espacios en los que se ha territorializado la política y donde se han implementado acciones contenidas en el Plan de Igualdad de Oportunidades para la Equidad de Género en relación con los derechos a la paz y convivencia con equidad de género, salud plena, trabajo en condiciones de igualdad y dignidad, educación con equidad, cultura libre de sexismo y hábitat y vivienda dignas. Estas acciones están referidas a los componentes de prevención, promoción, protección, atención y restablecimiento de los derechos de las mujeres en el distrito capital (Barreto, Barón y Buriticá, 2011).
} 
de Oportunidades para la Equidad de Género. De este modo, se lograron establecer siete CIO en diferentes localidades de Bogotá (cuadro 4).

\section{Cuadro 4. CIO en Bogotá}

\begin{tabular}{|l|c|l|}
\multicolumn{1}{c|}{$\begin{array}{c}\text { Casa de Igualdad de } \\
\text { Oportunidades Localidad }\end{array}$} & Fecha de Creación & \multicolumn{1}{|c|}{ Cobertura en los territorios } \\
\hline Casa Matriz La Candelaria & 2004 & Candelaria y Santa Fe \\
\hline CiO Ciudad Bolivar & 2005 & Ciudad Bolvar \\
\hline CiO Bosa & 2006 & Bosa \\
\hline CiO Suba & 2006 & Suba, \\
\hline CiO Sumapaz & 2006 & Sumapaz, Usme \\
\hline CiO Kennedy & 2007 & Kennedy y Antonio Nanino \\
\hline CiO Engativa & 2007 & Engativa.
\end{tabular}

Fuente: Secretaría Distrital de la Mujer (2012a).

Las CIO son una realización valiosa del Gobierno de Garzón, porque se convierten en espacios físicos, en los cuales las mujeres pueden interactuar y encontrar soluciones a sus requerimientos y necesidades.

En 2007, se crea la Gerencia de Mujer y Géneros en el Instituto Distrital de la Participación y de la Acción Comunal mediante el Acuerdo 02 de 2007, responsable hasta 2012 de la estrategia de territorialización a través de las CIO, que cubre el territorio distrital urbano y rural. Según una de las funcionarias entrevistadas, esta instancia se creó como una necesidad de no perder todo el capital humano acumulado en los territorios. Con el Gobierno de Samuel Moreno (2008-2012), se implementa el Programa Bogotá Positiva con las Mujeres y la Equidad de Género y se institucionaliza la Política Pública de Mujer y Género. Con el Gobierno de Gustavo Petro (2012-2016), se crea el Sector Administrativo Mujeres y la Secretaría Distrital de la Mujer mediante el Acuerdo 490 de 2012 y se expide el Decreto 001 de 2013 en el que "se establece la estructura organizacional y funciones de la Secretaría Distrital de la Mujer y se dictan otras disposiciones”.

En estos procesos, cabe mencionar la intervención de una serie de actores públicos y privados, que desempeñan un papel bastante importante en la construcción colectiva y puesta en marcha de la Política. Entre ellos, el Colectivo de Mujeres del Polo Democrático, las sindicalistas de la Federación Colombiana de Educadores, la Iniciativa de Mujeres por la Paz, la Comisión de Mujeres de la Asociación Distrital de Educadores y de la Central Unitaria de Trabajadores, el Grupo Mujer y Sociedad de la Universidad Nacional de Colombia, el Nodo de Mujeres Progresistas, la Mesa Diversa de Mujeres, y diversas organizaciones de mujeres de las veinte localidades.

En suma, el marco normativo, las acciones realizadas y la institucionalidad creada, reflejan la voluntad política de los gobernantes del distrito capital, y se convierten en la base fundamental para formular e implementar la Política Pública de Mujeres y Equidad de Género, como se muestra a continuación. 


\section{De la normativa a la práctica: los aciertos}

En el Gobierno del Alcalde Samuel Moreno, se pone en marcha formalmente la Política Pública de Mujeres y Equidad de Género mediante el Decreto 166 de 2010, con el objetivo de

reconocer, garantizar y restablecer los derechos de las mujeres, de manera que se modifiquen de forma progresiva y sostenible, las condiciones injustas y evitables de discriminación, subordinación y exclusión que enfrentan las mujeres en los ámbitos público y privado, promoviendo la igualdad real de oportunidades y la equidad de género en el distrito capital.

El artículo 8 del citado Decreto presenta las cuatro estrategias operativas de la Política: la transversalización, la territorialización, la corresponsabilidad y la comunicación, entendidas como el conjunto de decisiones y acciones político-administrativas del distrito capital. Este acto jurídico se torna en un instrumento legal, que debe ser acatado por los organismos distritales y la ciudadanía en general, y se constituye en un respaldo importante para la reivindicación de los derechos de las mujeres. En consecuencia, las estrategias desarrolladas marcan la hoja de ruta para la puesta en marcha de las acciones que permitan la transversalización de género como un tema relevante de la agenda distrital en todas las instancias operativas y administrativas.

Del mismo modo, la financiación de la Política prevé una asignación de los recursos de inversión del Distrito según la disponibilidad que se tenga. Se contempla la priorización de los presupuestos participativos sensibles al género con el concurso activo de los grupos, las organizaciones y las redes de las mujeres en las localidades del distrito capital. Cabe mencionar que el documento de la política no muestra un porcentaje de asignación presupuestal contundente que comprometa recursos significativos para su puesta en marcha.

La Política Pública de Mujeres y Equidad de Género se implementó en las alcaldías de Samuel Moreno y Gustavo Petro. Estos Gobiernos formalizaron los procesos interinstitucionales puestos en marcha desde 2004 y avanzaron en el cumplimiento de los objetivos propuestos para fortalecer la equidad de género en las diferentes instancias. En el Programa Bogotá Positiva con las Mujeres y la Equidad de Género del gobierno de Samuel Moreno (2008-2012), se contemplaba cumplir con ochenta acciones relacionadas con los derechos estipulados en el Plan de Igualdad de Oportunidades para la Equidad de Género. En este contexto, se destacan las siguientes realizaciones:

- La sensibilización a la ciudadanía sobre la violencia contra las mujeres.

- El posicionamiento del 25 de noviembre como Día Internacional de la no Violencia contra las Mujeres.

- La realización del seminario internacional "Ciudades seguras sin violencia contra las mujeres". 
- La realización de tres mesas “Diversas de Mujeres”, con participación de 300 mujeres por mesa.

- El establecimiento de Comités Locales de Mujer y Género, con resolución de 18 de ellos.

- La realización de la campaña "La ciudad se mueve por el derecho de las mujeres a un trabajo digno y en equidad".

- La conformación de redes de mujeres productoras y productivas.

- La realización de jornadas de salud para las mujeres, denominadas "Salud para las mujeres, vida para la ciudad”.

En cuanto a las CIO, en este Gobierno, la meta era poner en operación nueve CIO (cuadro 5), lo cual se cumplió porque todas estas CIO están en marcha actualmente. En este Gobierno, se reafirma la visibilización de las mujeres como ciudadanas de derechos y capacidades. Así lo indica una mujer que recibió el apoyo de las CIO:

La casa nos da posicionamiento político en la localidad. Empiezo a vincularme en todo lo que ocurre a nivel local en Teusaquillo y a nivel distrital, te atraviesa el cuerpo, la piel, te atraviesa la vida, en tu familia, con tus padres, hermanos, compañeros, con tus hijos, no te imaginas cómo la casa, sin ser feminista, te cambia, pienso que las mujeres tenemos que hacer cambios desde lo interno hacia afuera, cómo vamos a hablar de derechos cuando en lo privado nos están vulnerando (entrevista con Lilly Martínez, 30 de septiembre de 2013).

\section{Cuadro 5. CIO durante el Gobierno de Samuel Moreno}

\begin{tabular}{|c|c|c|}
\hline CTOILocalidad & Fecha de Creacion & Cobertura en los Territorios \\
\hline CIO Teusaquillo & 2008 & Teusaquilio, Chapinero \\
\hline CiO Rafael Uribe Uribe & 2008 & Rafael Unbe Unbe. \\
\hline CIO San Cristobal & 2008 & San Cristobal \\
\hline CIO Usaquen 23 & 2009 & Tunjuelito \\
\hline CIO Fontibon & 2009 & Fontibón \\
\hline ClO Puente Aranda & 2009 & Puente Aranda \\
\hline CiO San Cristobal & 2010 & San Cristobal \\
\hline ClO Barrios Unidos & 2010 & Bamos Unidos \\
\hline CIO Martires & 2011 & Martires \\
\hline
\end{tabular}

Fuente: Secretaría Distrital de la Mujer (2012)

En suma, con el Gobierno de Samuel Moreno, las estrategias en pro de las mujeres se direccionan no solo a ampliar la cobertura de las CIO, sino que, además, se dirigen a fortalecer las redes de mujeres para hacer seguimiento y control político a los procesos de dichas instancias y también de la Política.

Con el Gobierno de Gustavo Petro (2012-2016), nace el programa Bogotá Humana con Igualdad de Oportunidades y Equidad de Género para las Mujeres, cuya meta primordial es la creación y puesta en marcha de la Secretaría Distrital de la Mujer como 
entidad rectora de la Política Pública de Mujeres y Equidad de Género, mediante la expedición del Acuerdo 490 de 2012 y del Decreto 001 de 2013 en el que se establece su estructura organizacional y sus funciones. La creación de esta Secretaría es producto de la exigibilidad del movimiento social de mujeres ${ }^{11}$ frente a la garantía de derechos, del posicionamiento técnico de la gestión pública bajo el enfoque de derechos de las mujeres y de la voluntad política expuesta en la agenda de Gobierno de Bogotá Humana (Secretaría Distrital de la Mujer, 2013b, 2013c). Entre las acciones más relevantes que hasta el momento se han llevado a cabo con esta instancia, se destacan:

- El desarrollo de los Consejos de Seguridad para Mujeres en las localidades para analizar la situación de violencias contra ellas.

- La visibilización de la Secretaría Distrital de la Mujer como instancia de primer nivel ante los mecanismos de coordinación.

- La creación y consolidación de los Semilleros de Mujer y Géneros en instituciones educativas distritales.

- La vinculación de 26 servidoras y servidores públicos a procesos de sensibilización sobre el derecho de las mujeres a una vida libre de violencias.

- El reconocimiento al Plan de Igualdad de Oportunidades para la Equidad de Género en el distrito capital en 2012 como "buena práctica" por parte de la organización gubernamental New York City Global Partners.

Con relación a las CIO, en este Gobierno, se obtiene la primera mención especial por la experiencia denominada Estrategia de territorialización de la Política Pública de Mujeres y Equidad de Género mediante las Casas de Igualdad de Oportunidades para las Mujeres, dentro de la segunda edición de los Premios a las Mejores Prácticas Locales con Enfoque de Género, en septiembre de 2013, en Aguascalientes (México), mención otorgada a la Alcaldía Mayor de Bogotá en cabeza de la Secretaría Distrital de la Mujer (Secretaría Distrital de la Mujer, 2013a). Este reconocimiento a las CIO resalta a Bogotá como ciudad pionera en la promoción de la participación y representación de las mujeres en las CIO a lo largo de los nueve años de la implementación de la Política.

En consecuencia, dichos procesos adelantados en las CIO reflejan dinámicas que demuestran una relación estrecha entre las mujeres y sus territorios, teniendo en cuenta sus diversidades, necesidades y capacidades de acuerdo con la realidad de cada una de ellas y sus entornos. En palabras de una de las funcionarias de la Secretaría Distrital de la Mujer, los logros son evidentes, porque

\footnotetext{
${ }^{11}$ Proceso de incidencia realizado junto con el Consejo Consultivo de Mujeres, la Fundación de Apoyo Comunitario y diversas mujeres y organizaciones de mujeres participantes en la Alianza Pro-Secretaría de las Mujeres, con el apoyo de la Agencia de Cooperación Internacional.
} 
Entre abril y septiembre de este año han sido atendidas por esta Secretaría más de 7800 mujeres en procesos de acompañamiento sociojurídico especializado y en procesos de empoderamiento, que remiten a procesos de formación con las mujeres en el conocimiento de sus derechos, en pautas frente al relacionamiento con la institucionalidad, por ejemplo, para la exigibilidad y el control social, supone también la motivación para la participación política de las mujeres, entre otras iniciativas que se dinamizan a través de los procesos que se realizan fundamentalmente en las CIO (entrevista con Carlota Alméciga, 8 de octubre de 2013).

En síntesis, el Gobierno de Gustavo Petro logró avances significativos, como se ve con la creación y puesta en marcha de la Secretaría Distrital de la Mujer, que se torna en un instrumento muy relevante para la institucionalización de la Política. Del mismo modo, se destaca el incremento del equipo humano, administrativo y técnico que cubre la totalidad de las $\mathrm{CIO}$, que actualmente están funcionando en todo el distrito capital.

\section{Las fallas de la implementación}

Si bien la Política Pública de Mujeres y Equidad de Género presenta avances importantes en su implementación, también evidencia fallas a lo largo de su puesta en marcha, las cuales están relacionadas con el enfoque de la Política, la apropiación presupuestal, la difusión de información, la cobertura, el recurso humano y la ausencia de un sistema de evaluación y seguimiento. Uno de sus más grandes obstáculos se concentra en su etapa inicial y tiene que ver con el enfoque de la Política, porque paradójicamente las funcionarias del Distrito no asimilaron acertadamente el enfoque de "género" previsto en ella y lo abordaron, según algunos testimonios, como "embelecos feministas" (entrevista con Sandra Mojica, Bogotá, 18 de octubre 2013). Cabe señalar que con la puesta en marcha de las campañas de sensibilización que se realizaron en esta materia, tal limitación fue disminuyendo en el transcurso de los diferentes Gobiernos, hasta el punto de que las percepciones por parte de los funcionarios distritales son más acordes con los postulados de género y de derechos. Claro está que, para algunas de las funcionarias entrevistadas, sigue existiendo cierta apatía por parte de varios colegas que continúan tratando el tema de manera despectiva.

Otro de los inconvenientes que sale a relucir tiene que ver con los recursos económicos para la puesta en marcha de la Política. En los Gobiernos de Jaime Garzón y Samuel Moreno, no hay informes que den cuenta de los recursos que fueron asignados para ella; no obstante, se intuye que fueron pocos, no solo por el hecho de no encontrar reportes presupuestales, sino porque varias de las entrevistadas indicaron que los recursos previstos desde un comienzo han sido insignificantes y no suplen en efecto las necesidades reales de la política. En el Gobierno de Gustavo Petro, al contrario, sí se encuentra un reporte de la asignación presupuestal para la política con vigencia 
2012-2013 que asciende a \$2 906440 758, que es tan solo $0.03 \%$ del presupuesto de la Administración Distrital (Secretaría Distrital de la Mujer, 2013a, p. 21). Este aspecto se convierte en una falla bastante relevante para la implementación de la política y refleja la falta de compromiso en la incorporación del enfoque de género en los presupuestos de la agenda de los gobernantes, que, aunque tienen voluntad política para atender las problemáticas de las mujeres, no proporcionan los recursos suficientes para suplir todas las necesidades que demanda la política.

Una falla más que incide directamente con la cobertura de la población objetivo de la política está relacionada con las estrategias de comunicación y difusión, atribuidas, entre otras cosas, al costo que implica el uso de los medios masivos de comunicación en el país y a los escasos recursos con los que se ha contado. Así lo ratificó una de las funcionarias de la Secretaría Distrital de la Mujer:

Las comunicaciones son muy costosas, un minuto en televisión, no se habla de minutos de segundos, cada segundo son millones, la publicidad en medios masivos, revistas, periódicos de circulación nacional, son muy costosos, un minuto en radio es carísimo, y los recursos de la política pública siempre han sido muy escasos para invertir en eso, por tanto, no es algo que hayamos priorizados (entrevista con Sandra Mojica, 18 de octubre de 2013).

A lo anterior, se une la ausencia de informes, reportes o sistemas de información que den cuenta de indicadores que muestren el impacto real que la política está generando en la reivindicación de los derechos de las mujeres del distrito. Esto ha limitado el seguimiento de la política.

En cuanto al funcionamiento de las COI, se registran fallas que tienen que ver con su recurso humano, ${ }^{12}$ debido a que la planta de personal está vinculada por contratos de prestación de servicios, lo cual dificulta la continuidad de los procesos que se van trabajando dentro de estos lugares, ya que, en muchas ocasiones, al darse por terminado el contrato, se rompe el trabajo que los funcionarios vienen gestionando con las mujeres y con algunas instituciones, que conlleva una ruptura de los procesos y la desmotivación de las mujeres que deciden retirarse de las CIO, como lo expresó la referente local de la CIO de Usaquén:

Los que trabajamos en las CIO somos contratistas, eso limita un poco respecto de los procesos, porque es estar proyectando en términos del contrato, aun cuando tenemos plan de acción anual, y cuando tú terminas contrato, como que no te proyectas, porque puede que dures una semana o dos semanas. Desde el punto de vista de la

${ }^{12}$ Referente local, abogada, psicóloga y administrativo. 
realidad como contratista, ya sabes que dependes de los procesos organizativos y de la dinámica local y, en ese sentido, tú tienes un proceso y, si no lo terminas, rompe tu trabajo con las mujeres porque se deja de asistirlas (entrevista con Luz Dary Pedraza, 3 de octubre de 2013).

Lo anterior muestra las fallas institucionales y administrativas de la Política Pública de Mujeres y Equidad de Género, lo cual lleva a deducir que la implementación de la Política no corresponde completamente con lo trazado en su documento formal y, por tanto, esta no cumple de manera contundente con su propósito de reconocer, garantizar y restablecer los derechos de las mujeres de Bogotá.

\section{El modelo de implementación de la Política Pública de Mujeres y Equidad de Género}

Al contrastar los hallazgos empíricos de la implementación de la Política con los enfoques tradicionales top-down y bottom-up y el enfoque de gestión pública, se puede deducir que, en el primer caso, la política presenta rasgos de los dos enfoques, porque,por un lado, hay una marcada directriz jerárquica concebida desde el escenario internacional que baja al ámbito nacional y llega al Gobierno distrital, que acoge estos lineamientos y acondiciona instrumentos específicos para ponerla en marcha. Por otro lado, el proceso de implementación muestra desde su origen iniciativas concretas propiciadas por las mujeres del distrito, que coadyuvaron a la construcción y concertación de acciones y estrategias para abordar la problemática presentada. Esto demuestra que la Política Pública de Mujeres y Equidad de Género es un híbrido de estos dos enfoques, porque incorpora postulados de ambos, lo cual refleja, tal como lo plantea Roth (2009), la controversia existente entre los que creen más en el control, la planeación y la jerarquía y los que creen más bien en la concertación.

En el enfoque de gestión pública, se puede observar que la implementación de la Política Pública de Mujeres y Equidad de Género no cumple a cabalidad con todas las condiciones de éxito propuestas en él, que en teoría son el ideal para llevar a cabo una puesta en marcha exitosa. En el cuadro 6, se realiza una aproximación a estas condiciones a partir de lo encontrado en la implementación de la Política. Según esto, podemos decir que no se ha llevado a cabo una implementación completamente exitosa, porque no se cumplen dos de sus condiciones: una teoría sólida y una ley bien concebida. Aunque la política presenta una teoría y una ley formal y bien fundamentada, en la práctica, no se ha logrado una apropiación eficaz del enfoque conceptual de "derechos y de género" ni una completa sensibilización por parte de algunos funcionarios involucrados en el proceso. Tampoco se han cumplido a cabalidad con las estrategias y los objetivos trazados, en especial respecto de los recursos económicos y humanos. 
Cuadro 6. Condiciones de éxito para la implementación de la Política Pública de Mujeres y Equidad de Género del Distrito Capital

\begin{tabular}{|l|l|l|l|l|}
\hline Condiciones & Sí & No & \multicolumn{1}{|c|}{ Política Pública de Mujeres y Equidad de Género } \\
\hline solida & & Xna teoría & $\begin{array}{l}\text { Aunque es claro en el documento formal de la Política que el } \\
\text { enfoque que la guía es el "de derechos y de género", y que concibe } \\
\text { instrumentos claros para cumplir con los objetivos, también es } \\
\text { evidente que la apropiación del enfoque por parte de algunos } \\
\text { funcionarios no se ha dado plenamente, por lo cual no se genera } \\
\text { un cambio de comportamiento real en el momento de poner en } \\
\text { marcha la política y cumplir a cabalidad con sus objetivos. }\end{array}$ \\
\hline $\begin{array}{l}\text { Una ley bien } \\
\text { concebida }\end{array}$ & X & $\begin{array}{l}\text { Aunque la Política integra los seis aspectos que muestran una ley } \\
\text { bien concebida, estos no se cumplen a cabalidad, en especial en } \\
\text { la falta de recursos suficientes para ponerla en marcha y en la } \\
\text { ausencia de mecanismos claros para que los grupos organizados } \\
\text { puedan intervenir y hacer seguimiento a ella. }\end{array}$ \\
\hline $\begin{array}{l}\text { Responsables } \\
\text { capacitados y } \\
\text { comprometidos }\end{array}$ & $\mathrm{X}$ & $\begin{array}{l}\text { En los nueve años de la implementación de la Política, se observa } \\
\text { una voluntad política por parte de las diversas Administraciones } \\
\text { al incorporarla en sus respectivos planes de desarrollo. Asimismo, } \\
\text { algunos funcionarios han adquirido el compromiso en su } \\
\text { implementación, lo cual ha sido fortalecido con la creación de la } \\
\text { Secretaría Distrital de la Mujer, que se ha convertido en un espacio } \\
\text { para la construcción de iniciativas novedosas en pro de la Política. }\end{array}$ \\
\hline $\begin{array}{l}\text { Apoyo político } \\
\text { y social }\end{array}$ & $\mathrm{X}$ & $\begin{array}{l}\text { En la Política se observa el apoyo, por un lado, de las } \\
\text { organizaciones de mujeres, y otro, de las tres Administraciones } \\
\text { que la incorporaron en sus agendas y que han trabajado en la } \\
\text { consecución de sus propósitos. }\end{array}$ \\
\hline Entorno & Xavorable & $\begin{array}{l}\text { La Política, en el transcurso de su implementación, no se ha visto } \\
\text { afectada por el entorno ni por otras políticas que la alteren, porque } \\
\text { ha mantenido el apoyo político y normativo en los ámbitos nacional } \\
\text { e internacional. }\end{array}$ \\
\hline
\end{tabular}

Fuente: Elaboración propia a partir de Sabatier y Mazmanian (1979), citados por Roth (2000, pp. 114-115).

\section{Conclusiones}

Los eventos promovidos en el contexto internacional se convierten en el marco jurídico y de actuación de las políticas implementadas hacia las mujeres en los ámbitos nacional y distrital que sustentan la búsqueda de la reivindicación de los derechos de la población femenina y la igualdad de oportunidades entre mujeres y hombres. El diseño de la Política Pública de Mujeres y Equidad de Género del Distrito Capital es un reflejo de ello. Si bien muestra en su implementación avances y realizaciones importantes en el reconocimiento, la garantía y la restitución de los derechos de las mujeres y crea espacios relevantes para el fortalecimiento de los procesos de participación y organización de la población femenina, también indica fallas relacionadas con la dificultad de introducir el enfoque de género y derechos en los procesos de planeación e institucionalización de 
algunos sectores de la Administración distrital, así como dificultades en la asignación de presupuestos específicos para su puesta en marcha.

Se concluye que su proceso de implementación no ha sido completamente exitoso, porque no se ha dado una coherencia total entre los lineamientos trazados y lo ejecutado hasta el momento. Es decir, su formalismo no concuerda totalmente con la realidad.

\section{Referencias}

Alcaldía Mayor de Bogotá D. C. (2005). Plan de Igualdad de Oportunidades para la Equidad de Género. Recuperado de http://historico.equidadmujer.gov.co/mecanismos/ Documents/bogota/Politica\%2oPublica\%20Mujer\%2oGeneros\%2oBogota.pdf

Alcaldía Mayor de Bogotá D. C. (2007). Fechas de establecimiento de las Casas de Igualdad de Oportunidades. Bogotá.

Alcaldía Mayor de Bogotá D.C. Ley 1257 de 2008 (4 diciembre 2008).

Alcaldía Mayor de Bogotá D. C. Decreto 166 de 2010 (4 mayo 2010).

Alcaldía Mayor de Bogotá. Decreto oo1 de 2013 (3 de enero 2013).

Barreto, J., Barón, M. y Buriticá, M. (2011). Informe final comisiones temáticas. Bogotá. Congreso de la República. Ley 051 de 1981 (2 junio 1981).

Congreso de la República. Ley 248 de 1995 (29 diciembre 1995).

Congreso de la República. Ley 581 de 2000 (31 mayo 2000).

Congreso de la República. Ley 823 de 2003 (7 de julio de 2003).

Concejo de Bogotá D. C. Acuerdo 091 de 2003 (26 junio 2003).

Concejo de Bogotá D. C. Acuerdo 119 de 2004 (3 junio 2004).

Concejo de Bogotá D. C. Acuerdo 301 de 2007 (24 diciembre 2007).

Concejo de Bogotá D. C. Acuerdo 02 de 2007 (27 junio 2007).

Concejo de Bogotá D. C. Acuerdo 308 de 2008 (9 junio 2008).

Concejo de Bogotá D. C. Acuerdo 489 de 2012 (12 junio 2012).

Concejo de Bogotá D. C. Acuerdo 490 de 2012 (28 junio 2012).

DANE (2005). Censo General 2005. Recuperado de http://www.dane.gov.co/index. php/esp/poblacion-y-registros-vitales/censos/censo-2005

Fuentes Vásquez, L. Y. (2007). Las políticas públicas de mujer y género en Bogotá: tensiones y aciertos. Revista Colombiana de Sociología, 28. 
Instituto Colombiano de Bienestar Familiar. Decreto 2820 de 1974 (20 diciembre 1975). Instituto Colombiano de Bienestar Familiar. Decreto 1930 de 2013 (11 septiembre 2013). Meny, Y. y Thoenig, J.-C. (1992). Las políticas públicas. Barcelona: Ariel.

Muller, P. (2010). Las políticas públicas ( $3 \cdot^{\mathrm{a}}$ ed.). Bogotá: Universidad Externado de Colombia.

Muñoz Eraso, J. P. (2003). Aportes para el análisis de la política pública para el desarrollo de la mujer rural de 1994 en Colombia. Cuadernos de Desarrollo Rural, 50, 97-124.

ONU (2012). Informe Anual 2012: Los bonos en la mira, aporte y carga para las mujeres. Santiago de Chile: Observatorio de Igualdad de Género de América Latina y el Caribe.

Pineda Duque, J. A. (2007). Hacia una ciudad incluyente: género e indicadores sociales en Bogotá, Pre-til, 13, 29-47.

Presidente de la República de Colombia. Decreto 1182 de 1999 (20 junio 1999).

PNUD (s. f). Derechos humanos de las mujeres. Recuperado de http://www.americalatinagenera.org/es/index.php?option=com_contentyview=articleyid=367yIt emid $=334$

Roth Deubel, A.-N. (2009). Políticas públicas: formulación, implementación y evaluación. Bogotá: Ediciones Aurora.

Secretaría Distrital de la Mujer (2012a). Estrategia de territorialización de la Política Pública de Mujeres y Equidad de Género mediante las Casas de Igualdad de Oportunidades. Bogotá. Inédito.

Secretaría Distrital de la Mujer (2013a). Balance de la Política Pública de Mujeres y Equidad de Género: Avanzan las mujeres, avanza Bogotá. Bogotá.

Secretaría Distrital de la Mujer (2013b). Bogotá, ciudad metropolitana líder en la promoción de los derechos de las mujeres. Bogotá.

Secretaría Distrital de la Mujer (2013c). Plan estratégico. Bogotá.

Secretaría Distrital de la Mujer (2013d). Mujeres en cifras. Bogotá. 